\title{
Panning for Gold in Mould: Can we Increase the Odds for Fungal Genome Mining?
}

\author{
Cameron L. M. Gilchrist, Hang Li and Yit-Heng Chooi*
}

\begin{abstract}
Fungi are a rich source of bioactive small molecules. However, the large number of biosynthetic gene clusters (BGCs) encoding these molecules in their genomes suggests their biosynthetic potential is far greater than we previously appreciated. The mining of fungal genomes therefore holds great promise for the discovery of new chemical entities for pharmaceutical and agricultural applications. As more and more fungal genomes become available, the accompanying number of BGCs is quickly becoming unmanageable. Along with improving molecular genetic tools to accelerate the translation of BGCs to small molecules, we must devise strategies to prioritise BGCs most likely to encode the biosynthesis of novel small molecules and molecules with new or improved bioactivities or functions. In this perspective, we discuss existing and emerging strategies for prioritisation of BGCs to increase the odds of fruitful genome mining in fungi.
\end{abstract}

\section{Introduction}

It is undeniable that fungi and their small molecules have impacted human life in many ways. A number of fungal secondary metabolites (SMs; also known as natural products) - the antibiotic penicillin, cholesterol-lowering statins and the immunosuppressant cyclosporine, just to name a few - have transformed human medicine. On the flip side, fungi are known to produce mycotoxins that threaten the health of humans and livestock. Fungi are also arguably the major contributor to crop yield losses due to diseases, and some pose serious threats to immunocompromised individuals.

It has been known, even prior to the genomic era, that genes encoding the biosynthesis of SMs in fungi often exist in clusters, much like their bacterial counterparts. 1 However, when the first few filamentous fungal genomes were sequenced, the large number of biosynthetic gene clusters (BGCs) they possessed surprised the research community as they far exceeded the diversity of SMs perceived in these fungi based on previous natural product isolation studies. It became immediately obvious that many of the BGCs in these fungi remain silent under common laboratory growth environments due to their conditional expression. The influx of fungal genome sequencing data over the last decade has continued to reinforce this notion. A recent estimate from 581 fungal genomes suggests we may have only explored molecules encoded by $3 \%$ of predicted fungal BGCs. $2[3$ We have thus barely scratched the surface of the chemical diversity that fungi are capable of generating. For fungal plant and human

School of Molecular Sciences, The University of Western Australia, Perth, WA 6009, Australia. E-mail: yitheng.chooi@uwa.edu.au pathogen genomes, genome sequencing efforts have further exposed our lack of knowledge regarding the roles SMs could play in host-pathogen interactions, especially in light of recent gene expression studies of fungi within hosts. 4

With the revelation of more and more such 'biosynthetic dark matter' in fungi, the question then becomes how we can effectively tap into their hidden biosynthetic potential for bioactive molecule discovery. Approaches for translating BGCs in the genome to SMs have been reviewed elsewhere, but can be broadly categorised into manipulation of signalling pathways in the producing fungi and expression of BGCs in heterologous systems. Additionally, our ability to predict BGCs from fungal genomes is improving with the development of bioinformatics tools such as antiSMASH. 6 Despite these advances, a bottleneck remains due to the laborious and time-consuming natures of both the development of transformation procedures for non-model fungal species, and the heterologous reconstruction of BGCs; approaches that cannot keep up with the influx of genomic information ushered in by the advent of next generation sequencing technologies. Therefore, developing methods to effectively wade through this ocean of information is vital for such genome-based fungal natural product discovery going forward.

In this perspective, several existing and emerging ways for the prioritisation of fungal BGCs will be discussed. The primary interest in genome mining for fungal SMs has been its potential for the discovery of bioactive molecules for pharmaceutical and agrochemical applications. However, equally exciting are the opportunities to discover completely novel SM structural scaffolds, biosynthetic enzymes catalysing novel chemical reactions, new modes of action or SM targets, and molecules involved in impor- 
tant fungal biotic and abiotic interactions - all of which have the potential to translate into real world applications in the future.

\section{Love thy Neighbours}

A famous quote of Donald Rumsfeld's - the 'known knowns, unknown knowns and unknown unknowns' - was aptly used by Seyedsayamdost and Clardy 7 in their description of natural products and the research field. This is a lso a convenient way to categorise the BGCs in microbial genomes. BGCs linked to SMs can be considered 'known knowns,' while 'known unknowns' are those BGCs featuring known backbone biosynthetic enzymes, such as polyketide synthases (PKSs), nonribosomal peptide synthetases (NRPSs), terpene synthases (TSs) and prenyltransferases (PTases), which are easily predicted by software such as antiSMASH but yet to be linked to SMs. The 'unknown unknowns', on the other hand, are BGCs that are currently unrecognised by simple homology-based analysis due to our lack of knowledge concerning the type of biosynthetic genes involved - though such BGCs may well encode known molecules that have not yet been linked to BGCs. Mining of fungal genomes is typically focused on these 'known unknowns' and 'unknown unknowns.'

Perhaps the most straightforward way to explore the 'known unknowns' is to look no further than our existing drug leads. Many natural products exist in families of structurally-related compounds. Well known examples are the cholesterol-lowering statins, which include lovastatin, pravastatin, or mevastatin, and the antifungal echinocandins, which include echinocandin $B_{1}$, pneumocandin A, aculeacin, cryptocandin and mulundocandin. The structural similarities between such compounds are often reflected in the r elatedness of t heir B GCs. Targeting B GCs partially related to known BGCs may therefore be a good strategy for uncovering novel analogues of known bioactive compounds, which could possess improved pharmacological properties and help combat antibiotic resistance.

For such an approach to be successful, a highly curated database of characterised BGCs, linked to SM structures and other metadata, is crucial. One such example is the Minimum Information about a Biosynthetic Gene cluster (MIBiG) repository, 8 which allows for the deposition and retrieval of experimentally characterised BGC data. MIBiG has since been incorporated into antiSMASH as part of the KnownClusterBlast module, which analyses the similarity of predicted BGCs with homologous BGCs across many published genomes.

Another angle is to visualise BGC relatedness across many genomes as a network. In these analyses, BGCs are grouped into 'families' based on the presence of homologous genes and their similarities, which can cluster tightly given high conservation between BGCs, or more loosely when BGCs are more distant (Fig. 1). BGCs encoding compounds with varying levels of similarity to those encoding known molecules can then be readily identified based on the distances between members of families within a network. Such analysis has been performed with the Penicillium pan-genome, though it was restricted to conserved domains of backbone biosynthetic proteins, specifically PKSs and NRPSs.9 One such network analysis tool, Genome Neighbourhood Networks (GNNs), allows for large-scale prediction of enzyme function given their local genomic context. 10 This has recently been applied in the search for BGCs encoding novel enediyne analogues. ${ }^{11 / 12}$ Several tools are currently being developed to perform this analysis, such as the EFI-Genome Neighborhood Tool (EFI-GNT) 13 or BIG-SCAPE (https://git.wageningenur.nl/medema-group/BiGSCAPE, unpublished). Thus, this is a promising strategy for uncovering new members of a compound families; particularly those with useful bioactivities.

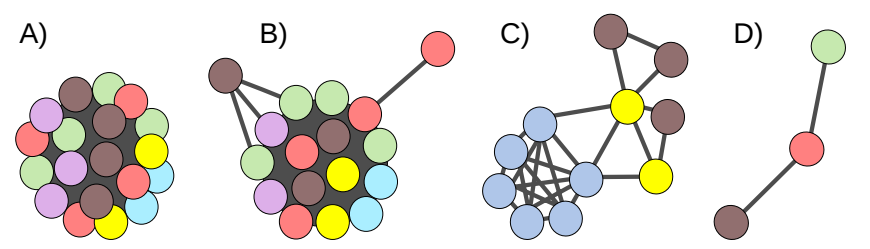

Fig. 1 Network analysis 'families' consisting of BGCs that are: A) highly conserved, B) conserved with some distant members, C) loosely related and D) distantly related and rare across taxa.

One may ask, then, why the BGC encoding the next cholesterollowering statin or echinocandin antifungal drug has not yet been found. So far, BGCs homologous to those encoding known clinical drugs have mainly been identified from fungal strains already known to produce compounds of the same family. Perhaps for this approach to truly be effective, a significantly larger number of fungal genomes is required, especially for those natural product families that are less distributed across taxa. This is illustrated in the mining of bacterial genomes for novel antitumor compounds related to enediynes. By surveying 3400 actinomycetes, Yan et al. 11 identified 28 distinct enediyne BGCs from 31 of 81 potential producer strains. The addition of these BGCs to a GNN containing all known enediyne BGCs made clear their substantial differences in enzymatic make-up, and their potential to produce new and diverse chemistry. This culminated in the identification of a new family of enediyne compounds, the tiancimycins, which showed potent cytotoxicity to cancer cell lines. More recently, this approach was extended to 11500 actinobacterial genomes, whereby 137 enediyne BGCs were uncovered, and a new anthraquinone-fused enediyne, yangpumicin A, was isolated. 12 This demonstrates the power of large-scale mining of pan-genomes for the discovery of novel analogues of known drug leads.

Fungal genomes are significantly larger than those of bacteria, meaning that there are relatively fewer that are publicly available. Nonetheless, fungal genome sequence databases are rapidly expanding, making this type of analysis increasingly viable. There are currently at least 3620 fungal genomes available across NCBI and JGI MycoCosm alone. This will only increase thanks to initiatives such as the 1000 Fungal Genomes Project (http://1000.fungalgenomes.org). Even at a smaller scale, we see a number of examples where uncovering the BGCs of known bioactive compounds opened up opportunities to discover related analogues, such as with mycophenolic acid. 14 Continued mapping of promising structural leads to their BGCs will generate new avenues for further exploration. 


\section{Finding the Oddballs}

Natural products with rare or novel structural scaffolds could possess new modes of action and lay the basis for new drug leads. They also often serve as inspiration in the development of new reactions for organic synthesis. Additionally, such rare scaffolds could indicate the involvement of novel enzymes in their biosynthesis. In the case of antibiotics, novel scaffolds with new modes of action are needed as analogues of existing scaffolds are more prone to resistance generation. Continuing to search for 'oddballs' could therefore be a very rewarding endeavour.
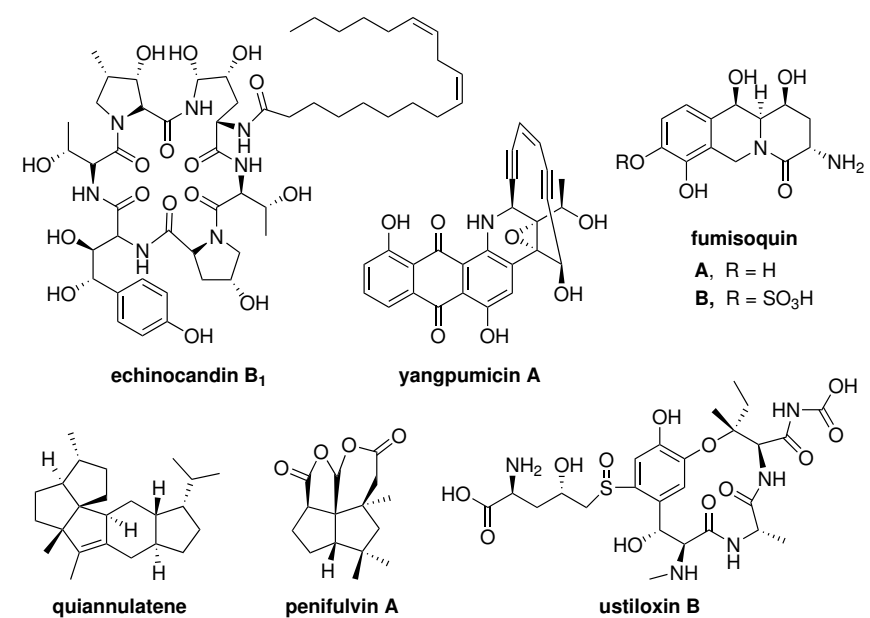

angpumicin A

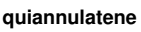

penifulvin $\mathrm{A}$
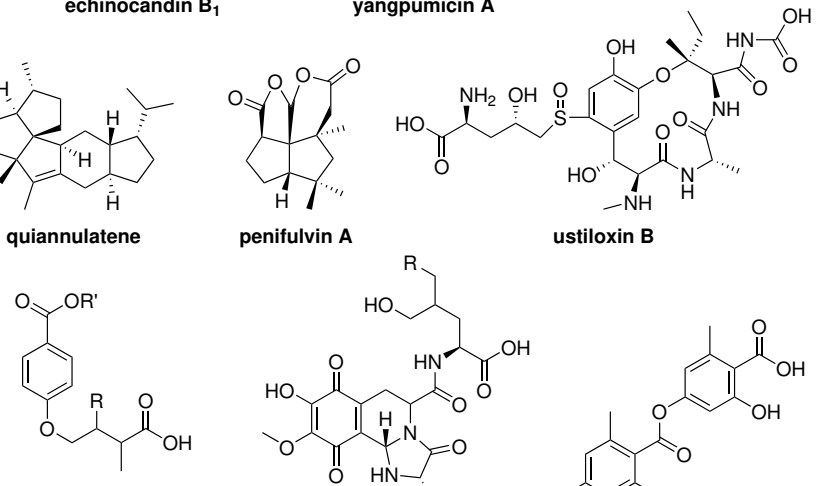

sporovexin

A, $\mathrm{R}=\mathrm{H} \quad \mathrm{R}^{\prime}=\mathrm{OH}$

B, $\mathrm{R}=\mathrm{OH} \quad \mathrm{R}^{\prime}=\mathrm{OH}$

C, $\mathrm{R}=\mathrm{H} \mathrm{R}^{\prime}=\mathrm{NHC}_{2} \mathrm{H}_{4} \mathrm{CO}_{2} \mathrm{H}$

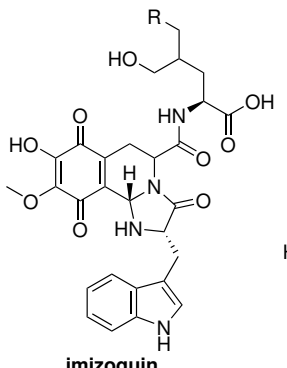

ustiloxin B

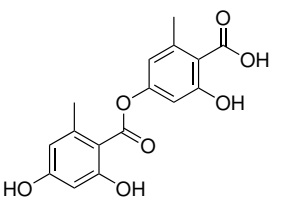

lecanoric acid
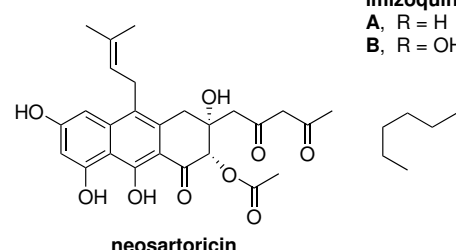

B, $\mathrm{R}=\mathrm{OH}$

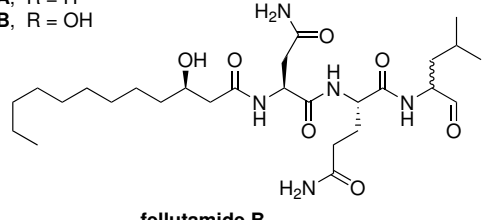

fellutamide $B$

Fig. 2 Representative structures mentioned in this perspective.

Network analysis of BGC similarity across many genomes, as discussed above, will allow us to sift through the 'known unknowns' while excluding those with significant similarities to 'known knowns.' Such analyses will enable the rapid identification of those BGCs unrelated to known BGCs and unique only to very few species (Fig. 1d), which could lead to the discovery of new classes of compounds. Another good starting point is to search for genes that encode proteins with unusual domain architectures, or that are only distantly related to known backbone biosynthetic genes. For example, Hang et al. $\frac{15}{15}$ searched 581 ascomycete and basidiomycete genomes and identified a novel class of highly-reducing PKSs harbouring a C-terminal domain with strong homology to carnitine O-acyltransferase (cAT). Though the final product of the BGC was not identified, they demonstrated that the cAT domain confers a novel mode of product release via esterification with polyalcohol nucleophiles, and is capable of recapturing the product to afford a second methylation and generate an $\alpha, \alpha$-gem-dimethyl product. Similarly, a series of novel NRPSs have been observed which novel domain architectures result in novel products. Baccile et al. $\frac{16}{16}$ identified a BGC within Aspergillus fumigatus containing $f s q$, which encodes a small NRPSlike protein which lacks the canonical condensation (C) domain, and is responsible for the production of several novel isoquinoline alkaloids named the fumisoquins. Similar genome mining efforts for unconventional terpene biosynthetic enzymes have also been fruitful. For example, Okada et al. 17 identified a sesterterpene synthase, EvQS, containing a novel terpene cyclase domain not belonging to any previously proposed phylogenetic clade. EvQS possesses a novel cyclization mode responsible for the production of the sesterterpene quiannulatene, which contains a highly congested carbon skeleton.

Unfortunately, this approach does not extend to the 'unknown unknowns,' which do not contain conventional backbone biosynthetic genes. Several tools have been developed in order to detect these BGCs, such as ClusterFinder 18 and MIDDAS-M. 19 ClusterFinder, available as part of antiSMASH, employs a hidden Markov model to probabilistically predict BGC-like regions in the genome based on frequencies of protein family domains observed in curated BGC and non-BGC training sets, and has been shown to successfully detect BGCs of unknown class. ${ }^{18}$ ClusterFinder works on the assumption that though the biosynthesis of unknown compounds may differ greatly to known compounds, key reactions still require similar enzyme families. 20 On the other hand, MIDDAS-M combines both genomic and transcriptomic data, and predicts BGCs by analysing the transcriptional co-regulation of neighbouring genes. Use of this tool enabled the identification of a BGC encoding the ribosomally synthesized and post-translationally modified peptide (RiPP) ustiloxin B in Aspergillus flavus. 19 The identification of 'unknown unknown' BGCs could have profound implications as it opens up new doors for discovery of new families of compounds. For instance, the discovery of the BGC encoding ustiloxin spurred the exciting discovery that such RiPP pathways are widespread across the ascomycetes and basidiomycetes. $21 / 22$

\section{Following the Trail}

Chemical ecology has served as inspiration in the search for SMs with bioactivities against specific organisms. For example, numerous phytotoxic compounds, which hold promise as potential herbicide leads, have been isolated from fungal pathogens of weeds. ${ }^{23}$ Similarly, antifungal compounds have been isolated from mycoparasitic or fungicolous fungi, such as penifulvin A Penicillium griseofulvum. 24 Another example is the insecticidal mycotoxin beauvericin isolated from Beauveria bassiana, a known insect pathogen. $\frac{25}{25}$ Study of coprophilous fungi, which compete against one another on dung, has revealed several compounds with antifungal and antibacterial activities such as the sporovexins A-C. ${ }^{26}$ However, many BGCs in these fungi could remain silent in laboratory culture conditions, only being expressed when the 
organism is being challenged in their native environment. These conditionally-expressed, ecologically-relevant BGCs can be narrowed down using transcriptomics and comparative genomics tools; such an approach has previously been referred to as chemical ecogenomics. ${ }^{4}$ For example, a silent BGC from the wheat pathogen Parastagonospora nodorum, upregulated during the late stage of wheat leaf infection, is responsible for the production of the light-activated phytotoxin elsinochrome $\mathrm{C}$, which has been shown to be involved in wheat virulance. 27 In another example, use of comparative genomics analysis revealed BGCs conserved across multiple animal and human-associated pathogens, not present in related non-pathogenic species of their corresponding genus. Activation of these pathways in Neosartorya fischeri and A. fumigatus, as well as heterologous expression of representative pathways from Trichophyton and Arthroderma dermatophytes, led to the discovery of the novel immunosuppressive neosartoricins. 28,29

Co-culture of microbial species, be it bacteria-fungi or fungifungi, has been successful in activating the production of cryptic natural products. $\frac{30,31}{3}$ For example, co-culture of Aspergillus nidulans with soil-dwelling actinomycetes was shown to activate fungal secondary metabolism genes, and enabled the production of several compounds including the lichen metabolite lecanoric acid. 32 Similarly, co-culture of naturally co-occurring, wood-decaying basidiomycetes resulted in the discovery of two novel bioactive xylosides. 33 However, in practice, it can be difficult to pinpoint which components of these interactions are actually responsible for the production of isolated compounds, and there could be additional complication in scaling up co-cultures (e.g. differences in growth rates). In such instances, transcriptomics could be used to identify candidate BGCs, which could then be linked to the metabolites (e.g. by pathway-specific activation or heterologous expression) in the absence of the requisite interaction partner. A recent study employed this approach: comparison of transcriptomic data revealed a putative BGC in A. flavus $\mathrm{d}$ ownregulated b y b acterial 1 ipopeptides; overexpression of a pathway-specific transcription factor in this B GC led to the isolation of the alkaloids imizoquins, which suppress bacterial growth. 34 The study revealed that SMs are involved in mediating the antagonism between fungi and bacteria, and that such antagonistic relationships could be exploited for genome mining of novel antibacterial and antifungal compounds.

\section{The Secret Resistance}

Organisms often encode self-resistance mechanisms for the bioactive SMs they produce to prevent suicide. Such mechanisms are often encoded by the very BGC responsible for the biosynthesis of the SM. 1 1 This can manifest itself as a duplicate of the SM target embedded in the BGC (Fig. 3). For example, the BGC encoding the biosynthesis of lovastatin, which blocks cholesterol synthesis via inhibition of HMG-CoA reductase, encodes a duplicated HMG-CoA reductase gene. 35,36 It remains unclear whether some of these duplicated target genes encode proteins that are resistant to the SM inhibition or merely provide excess targets within cells as a way to confer resistance to the organism. Nonetheless, this provide a means to uncover BGCs encoding SMs with known targets. Indeed, the BGC encoding mycophenolic acid, an immunosuppressive compound which inhibits IMP dehydrogenase (IMPDH), was localised by searching for duplicated IMPDH genes in P. brevicompactum. 37 Similarly, the BGC encoding fumagillin, which blocks blood vessel formation by inhibiting methioninine aminopeptidases, was uncovered due to the presence of duplicated methionine aminopeptidase- 1 and -2 genes found in the vicinity of a PKS gene predicted to be required for formation of fumagillin's decatetraenedioic acid moeity. ${ }^{38[39}$ The identification of the fumagillin BGC uncovered a novel family of terpene cyclases in fungi. 38

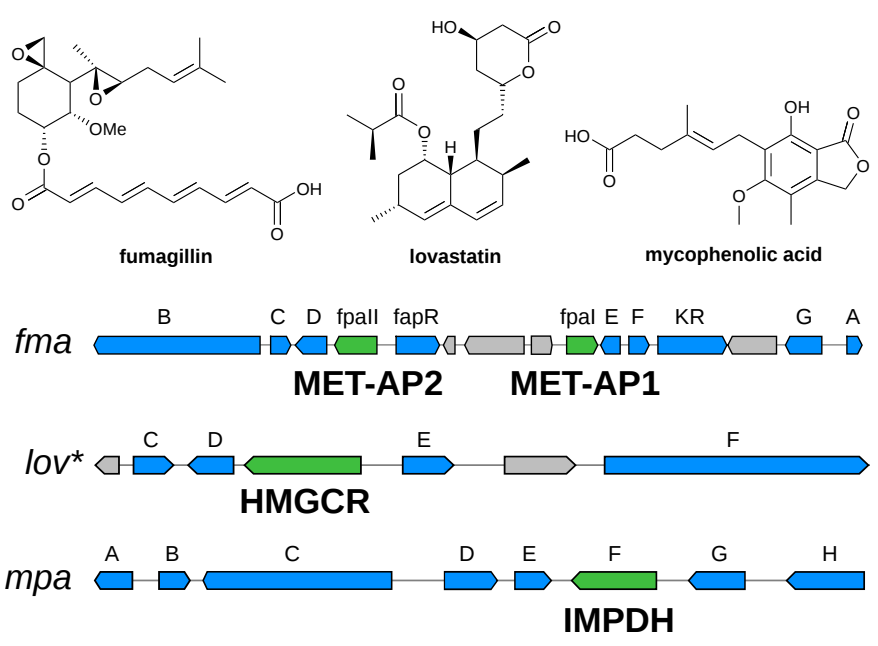

Fig. 3 Biosynthetic gene clusters encoding biosynthesis of fumagillin (fma), lovastatin (lov, partial) and mycophenolic acid (mpa). Boxed arrows in green, resistant duplicate copies of the compound target; blue, biosynthetic genes; grey, uncharacterised or unrelated to biosynthesis. HMGCR = HMG-CoA reductase, IMPDH = inosine-5'-monophosphate dehydrogenase, MET-AP = methionine aminopeptidase.

On the same token, we could potentially infer the target or mode of action of the SMs encoded by a BGC given the presence of a resistance target within that BGC. This has since formed the basis of a genome mining approach where BGCs containing non-biosynthetic, resistance-related genes are prioritized. Initial implementations of this approach have been limited in scope, either searching for, or chancing upon, specific drug targets of interest. For example, the previously uncharacterized inp BGC in $A$. nidulans contains a gene, inpE, encoding a duplicated proteasome subunit, suggesting a potential resistant target. ${ }^{40}$ Subsequent expression of this BGC resulted in production of the proteasome inhibitor fellutamide B. The proteasome is a known anticancer target, and proteasome inhibitors such as Bortezomib and Carfilzomib have recently been approved by the FDA. This highlights that this approach has significant potential for the discovery of SMs with desired modes of action.

Though foraging for BGCs encoding duplicates of specific validated or promising drug targets can be useful, a way to indiscriminately identify all duplicate resistant targets from entire genomes is greatly desirable. Such a pipeline, referred to as resistant target-directed genome mining, has just been demonstrated in the marine actinomycete genus Salinispora. ${ }^{41}$ Clusters of orthol- 
ogous groups (COGs) were first identified in 86 Salinispora strains to establish both pan- and core-genomes. The pan-genome was then queried for duplicates of core-genome COGs associated with natural product BGCs. Finally, a list of promising targets is generated by filtering these duplicates for those with resistance-related functions. As of writing, an automated pipeline to perform similar analyses has been made available named Antibiotic Resistant Targets Seeker (ARTS), though it has thus far only been implemented for bacteria. $\frac{42}{2}$ Nonetheless, one can imagine the added value of applying this to fungi, given they are eukaryotic organisms sharing many homologous targets with animals and plants. As such, the SMs encoded by these duplicated target-containing BGCs may serve as leads for human and veterinary drug as well as herbicide development. However, a potential obstacle that we would encounter for such an approach for fungi is that many duplicated COGs may be primary metabolite pathway genes that have been neofunctionalised to have a biosynthetic role in the BGC, which could be difficult to differentiate from a resistant target. For example, the duplicated isopropyl-malate synthase gene hty $A$ in the echinocandin producer Emericella rugulosa has been shown to be involved in biosynthesis of the non-proteinogenic amino acid homotyrosine required for echinocandin biosynthesis. ${ }^{43}$ A potential workaround would be to generate a list of duplicate COGs found in BGCs, followed by further prioritisation based on their potential applications as drug or herbicidal targets. Given that the final SM product of the BGC is likely to be bound to the resistant target (either at the active site or allosterically), whereas a biosynthetic enzyme would act on an intermediate, it might be possible to predict the likelihood of a duplicated gene encoding a resistant target or biosynthetic enzyme via protein modelling and docking analysis in the future, when we better understand how duplicate targets confer resistance.

\section{Future Outlook}

Continuous improvements in the computational and molecular tools we use to predict BGCs and translate them into molecules will be key to the success of future genome mining efforts. At the same time, we should not overlook the ongoing endeavor to connect SMs to their BGCs, as it will help expand our knowledge base on SM-BGC relationships As far as computational strategies are concerned, perhaps the holy grail is that one day we are not only able to predict the structure of the molecules encoded by BGCs, but also the function or bioactivities of these molecules. As more and more BGCs are characterised, and more biochemistry unveiled, our ability to predict the structure of compounds is improving. Prediction of potential function is more challenging; however, there are strategies we can use. The relatedness of BGCs to those encoding known bioactive molecules may provide us with clues as to their bioactivities; we can also get an idea of function given the ecology of the organism and the expression context of it's BGCs. Perhaps the most exciting strategy is the resistant target-directed genome mining, which can indicate a potential resistant target and mode of action of a molecule; though these targets are not present in every BGC. Going into the future, a combination of these strategies will likely prove vital in generating leads for pharmaceutical and agricultural applications.

\section{Acknowledgements}

C.L.M.G and H.L. are supported by an Australian Government Research Training Program PhD Scholarship and an International Postgraduate Research Scholarship, respectively. Y.-H.C and the research of his group in this area is supported by an Australian Research Council (ARC) Future Fellowship and an ARC Discovery Project grant (DP170100228). We thank Dr. Ralph Cacho (University of Washington) for helpful discussion.

\section{References}

1 N. P. Keller, Nat. Chem. Biol., 2015, 11, 671-677.

2 Y. F. Li, K. J. S. Tsai, C. J. B. Harvey, J. J. Li, B. E. Ary, E. E. Berlew, B. L. Boehman, D. M. Findley, A. G. Friant, C. A. Gardner, M. P. Gould, J. H. Ha, B. K. Lilley, E. L. McKinstry, S. Nawal, R. C. Parry, K. W. Rothchild, S. D. Silbert, M. D. Tentilucci, A. M. Thurston, R. B. Wai, Y. Yoon, R. S. Aiyar, M. H. Medema, M. E. Hillenmeyer and L. K. Charkoudian, Fungal Genet. Biol., 2016, 89, 18-28.

3 C. T. Walsh and Y. Tang, Natural Product Biosynthesis, The Royal Society of Chemistry, Croydon, 2017, pp. 696-700.

4 Y.-H. Chooi and P. S. Solomon, Front. Microbiol., 2014, 5, 640.

5 D. J. Mattern, V. Valiante, S. E. Unkles and A. A. Brakhage, Front. Microbiol., 2015, 6, 775.

6 K. Blin, T. Wolf, M. G. Chevrette, X. Lu, C. J. Schwalen, S. A. Kautsar, H. G. Suarez Duran, E. L. C. de los Santos, H. U. Kim, M. Nave, J. S. Dickschat, D. A. Mitchell, E. Shelest, R. Breitling, E. Takano, S. Y. Lee, T. Weber and M. H. Medema, Nucleic Acids Res., 2017, 1-6.

7 M. R. Seyedsayamdost and J. Clardy, ACS Synth. Biol., 2014, 3, 745-747.

8 M. H. Medema, R. Kottmann, P. Yilmaz, M. Cummings, J. B. Biggins, K. Blin, I. de Bruijn, Y.-H. Chooi, J. Claesen, R. C. Coates, P. Cruz-Morales, S. Duddela, S. Düsterhus, D. J. Edwards, D. P. Fewer, N. Garg, C. Geiger, J. P. Gomez-Escribano, A. Greule, M. Hadjithomas, A. S. Haines, E. J. N. Helfrich, M. L. Hillwig, K. Ishida, A. C. Jones, C. S. Jones, K. Jungmann, C. Kegler, H. U. Kim, P. Kötter, D. Krug, J. Masschelein, A. V. Melnik, S. M. Mantovani, E. A. Monroe, M. Moore, N. Moss, H.-W. Nützmann, G. Pan, A. Pati, D. Petras, F. J. Reen, F. Rosconi, Z. Rui, Z. Tian, N. J. Tobias, Y. Tsunematsu, P. Wiemann, E. Wyckoff, X. Yan, G. Yim, F. Yu, Y. Xie, B. Aigle, A. K. Apel, C. J. Balibar, E. P. Balskus, F. BaronaGómez, A. Bechthold, H. B. Bode, R. Borriss, S. F. Brady, A. A. Brakhage, P. Caffrey, Y.-Q. Cheng, J. Clardy, R. J. Cox, R. De Mot, S. Donadio, M. S. Donia, W. A. van der Donk, P. C. Dorrestein, S. Doyle, A. J. M. Driessen, M. Ehling-Schulz, K.-D. Entian, M. A. Fischbach, L. Gerwick, W. H. Gerwick, H. Gross, B. Gust, C. Hertweck, M. Höfte, S. E. Jensen, J. Ju, L. Katz, L. Kaysser, J. L. Klassen, N. P. Keller, J. Kormanec, O. P. Kuipers, T. Kuzuyama, N. C. Kyrpides, H.-J. Kwon, S. Lautru, R. Lavigne, C. Y. Lee, B. Linquan, X. Liu, W. Liu, A. Luzhetskyy, T. Mahmud, Y. Mast, C. Méndez, M. Metsä-Ketelä, J. Micklefield, D. A. Mitchell, B. S. Moore, L. M. Moreira, R. Müller, B. A. Neilan, M. Nett, J. Nielsen, F. O'Gara, H. Oikawa, A. Osbourn, M. S. Osburne, B. Ostash, S. M. Payne, J.-L. Pern- 
odet, M. Petricek, J. Piel, O. Ploux, J. M. Raaijmakers, J. A. Salas, E. K. Schmitt, B. Scott, R. F. Seipke, B. Shen, D. H. Sherman, K. Sivonen, M. J. Smanski, M. Sosio, E. Stegmann, R. D. Süssmuth, K. Tahlan, C. M. Thomas, Y. Tang, A. W. Truman, M. Viaud, J. D. Walton, C. T. Walsh, T. Weber, G. P. van Wezel, B. Wilkinson, J. M. Willey, W. Wohlleben, G. D. Wright, N. Ziemert, C. Zhang, S. B. Zotchev, R. Breitling, E. Takano and F. O. Glöckner, Nat. Chem. Biol., 2015, 11, 625-631.

9 J. C. Nielsen, S. Grijseels, S. Prigent, B. Ji, J. Dainat, K. F. Nielsen, J. C. Frisvad, M. Workman and J. C. Nielsen, Nat. Microbiol., 2017, 2, 17044.

10 S. Zhao, A. Sakai, X. Zhang, M. W. Vetting, R. Kumar, B. Hillerich, B. San Francisco, J. Solbiati, A. Steves, S. Brown, E. Akiva, A. Barber, R. D. Seidel, P. C. Babbitt, S. C. Almo, J. A. Gerlt and M. P. Jacobson, Elife, 2014, 3, e03275.

11 X. Yan, H. Ge, T. Huang, Hindra, D. Yang, Q. Teng, I. Crnovčić, X. Li, J. D. Rudolf, J. R. Lohman, Y. Gansemans, X. Zhu, Y. Huang, L.-X. Zhao, Y. Jiang, F. Van Nieuwerburgh, C. Rader, Y. Duan and B. Shen, MBio, 2016, 7, e02104-16.

12 X. Yan, J.-J. Chen, A. Adhikari, D. Yang, I. Crnovcic, N. Wang, C.-Y. Chang, C. Rader and B. Shen, Org. Lett., 2017, 19, 61926195.

13 J. A. Gerlt, Biochemistry, 2017, 56, 4293-4308.

14 B. G. Hansen, E. Mnich, K. F. Nielsen, J. B. Nielsen, M. T. Nielsen, U. H. Mortensen, T. O. Larsen and K. R. Patil, Appl. Environ. Microbiol., 2012, 78, 4908-4913.

15 L. Hang, M.-C. Tang, C. J. B. Harvey, C. G. Page, J. Li, Y.-S. Hung, N. Liu, M. E. Hillenmeyer and Y. Tang, Angew. Chemie Int. Ed., 2017, 56, 9556-9560.

16 J. A. Baccile, J. E. Spraker, H. H. Le, E. Brandenburger, C. Gomez, J. W. Bok, J. Macheleidt, A. A. Brakhage, D. Hoffmeister, N. P. Keller and F. C. Schroeder, Nat. Chem. Biol., 2016, 12, 419-424.

17 M. Okada, Y. Matsuda, T. Mitsuhashi, S. Hoshino, T. Mori, K. Nakagawa, Z. Quan, B. Qin, H. Zhang, F. Hayashi, H. Kawaide and I. Abe, J. Am. Chem. Soc., 2016, 138, 1001110018.

18 P. Cimermancic, M. H. Medema, J. Claesen, K. Kurita, L. C. Wieland Brown, K. Mavrommatis, A. Pati, P. A. Godfrey, M. Koehrsen, J. Clardy, B. W. Birren, E. Takano, A. Sali, R. G. Linington and M. A. Fischbach, Cell, 2014, 158, 412-421.

19 M. Umemura, H. Koike and M. Machida, Front. Microbiol., 2015, 6, 1-14.

20 T. Weber, K. Blin, S. Duddela, D. Krug, H. U. Kim, R. Bruccoleri, S. Y. Lee, M. A. Fischbach, R. Müller, W. Wohlleben, R. Breitling, E. Takano and M. H. Medema, Nucleic Acids Res., 2015, 43, W237-W243.

21 W. Ding, W.-Q. Liu, Y. Jia, Y. Li, W. A. van der Donk and Q. Zhang, Proc. Natl. Acad. Sci., 2016, 113, 3521-3526.

22 N. Nagano, M. Umemura, M. Izumikawa, J. Kawano, T. Ishii, M. Kikuchi, K. Tomii, T. Kumagai, A. Yoshimi, M. Machida, K. Abe, K. Shin-ya and K. Asai, Fungal Genet. Biol., 2016, 86, 58-70.
23 A. Cimmino, M. Masi, M. Evidente, S. Superchi and A. Evidente, Nat. Prod. Rep., 2015, 32, 1629-1653.

24 S. H. Shim, D. C. Swenson, J. B. Gloer, P. F. Dowd and D. T. Wicklow, Org. Lett., 2006, 8, 1225-1228.

25 R. Hamill, C. Higgens, H. Boaz and M. Gorman, Tetrahedron Lett., 1969, 10, 4255-4258.

26 A. G. Soman, J. B. Gloer, B. Koster and D. Malloch, J. Nat. Prod., 1999, 62, 659-661.

27 Y.-H. Chooi, G. Zhang, J. Hu, M. J. Muria-Gonzalez, P. N. Tran, A. Pettitt, A. G. Maier, R. A. Barrow and P. S. Solomon, Environ. Microbiol., 2017, 19, 1975-1986.

28 Y.-H. Chooi, J. Fang, H. Liu, S. G. Filler, P. Wang and Y. Tang, Org. Lett., 2013, 15, 780-783.

29 W.-B. Yin, Y.-H. Chooi, A. R. Smith, R. A. Cacho, Y. Hu, T. C. White and Y. Tang, ACS Synth. Biol., 2013, 2, 629-634.

30 A. Marmann, A. Aly, W. Lin, B. Wang and P. Proksch, Mar. Drugs, 2014, 12, 1043-1065.

31 T. Netzker, J. Fischer, J. Weber, D. J. Mattern, C. C. König, V. Valiante, V. Schroeckh and A. A. Brakhage, Front. Microbiol., 2015, 6, 1-13.

32 V. Schroeckh, K. Scherlach, H.-W. Nutzmann, E. Shelest, W. Schmidt-Heck, J. Schuemann, K. Martin, C. Hertweck and A. A. Brakhage, Proc. Natl. Acad. Sci., 2009, 106, 1455814563.

33 L. Yao, L.-P. Zhu, X.-Y. Xu, L.-L. Tan, M. Sadilek, H. Fan, B. Hu, X.-T. Shen, J. Yang, B. Qiao and S. Yang, Sci. Rep., 2016, 6, 33237.

34 S. Khalid, J. A. Baccile, J. E. Spraker, J. Tannous, M. Imran, F. C. Schroeder and N. P. Keller, ACS Chem. Biol., 2018, 13, 171-179.

35 J. Kennedy, Science, 1999, 284, 1368-1372.

36 Y. Abe, T. Suzuki, T. Mizuno, C. Ono, K. Iwamoto, M. Hosobuchi and H. Yoshikawa, Mol. Genet. Genomics, 2002, 268, 130-137.

37 T. B. Regueira, K. R. Kildegaard, B. G. Hansen, U. H. Mortensen, C. Hertweck and J. Nielsen, Appl. Environ. Microbiol., 2011, 77, 3035-3043.

38 H.-C. Lin, Y.-H. Chooi, S. Dhingra, W. Xu, A. M. Calvo and Y. Tang, J. Am. Chem. Soc., 2013, 135, 4616-4619.

39 P. Wiemann, C.-J. Guo, J. M. Palmer, R. Sekonyela, C. C. C. Wang and N. P. Keller, Proc. Natl. Acad. Sci., 2013, 110, 17065-17070.

40 H.-H. Yeh, M. Ahuja, Y.-M. Chiang, C. E. Oakley, S. Moore, O. Yoon, H. Hajovsky, J.-W. Bok, N. P. Keller, C. C. C. Wang and B. R. Oakley, ACS Chem. Biol., 2016, 11, 2275-2284.

41 X. Tang, J. Li, N. Millán-Aguiñaga, J. J. Zhang, E. C. O’Neill, J. A. Ugalde, P. R. Jensen, S. M. Mantovani and B. S. Moore, ACS Chem. Biol., 2015, 10, 2841-2849.

42 M. Alanjary, B. Kronmiller, M. Adamek, K. Blin, T. Weber, D. Huson, B. Philmus and N. Ziemert, Nucleic Acids Res., 2017, 1-7.

43 R. A. Cacho, W. Jiang, Y.-H. Chooi, C. T. Walsh and Y. Tang, J. Am. Chem. Soc., 2012, 134, 16781-16790. 